\title{
Mild encephalopathy with reversible splenial lesion associated with SARS-CoV-2 infection: A case report
}

\author{
${ }^{1}$ Fettah Eren $M D,{ }^{1}$ Gokhan Ozdemir $M D,{ }^{1}$ Omer Faruk Ildiz $M D,{ }^{2}$ Dilek Ergun $M D$, \\ ${ }^{1}$ Serefnur Ozturk $M D$
}

${ }^{1}$ Department of Neurology, ${ }^{2}$ Department of Chest Diseases, Selcuk University, School of Medicine, Konya, Turkey

\begin{abstract}
Mild encephalopathy with reversible splenial lesion (MERS) is characterized with a reversible lesion in the splenium of the corpus callosum. It has been defined as a neuro-radiologic syndrome associated with viral infections. In addition, this lesion may related with epileptic seizures, antiepileptic drugs or metabolic disturbances. We presented a patient with MERS associated with severe acute respiratory syndrome coronavirus 2 (SARS-COV-2). Encephalopathy and psychiatric symptoms were the major initial clinical symptoms. Brain magnetic resonance imaging showed complete resolution of the corpus callosum lesion. The symptoms of patient recovered completely. Complete resolution of corpus callosum lesion and recovery of encephalopathy associated with SARS-COV-2 were similar to previous cases.
\end{abstract}

Keywords: Coronavirus; corpus callosum; SARS-COV-2; encephalopathy.

\section{INTRODUCTION}

Severe acute respiratory syndrome coronavirus 2 (SARS-COV-2) infection presents mainly with respiratory symptoms. Besides the respiratory symptoms, neurological disorders consists of cerebrovascular disease, hemophagocytic lymphohistiocytosis, acute necrotizing hemorrhagic encephalopathy and Guillain-Barre syndrome. In rare cases, mild encephalitis/ encephalopathy with reversible splenial lesion (MERS) was reported associated with SARSCOV-2 infection. ${ }^{1-3}$

MERS is characterized with a reversible lesion in the splenium of the corpus callosum. This lesion may related with epileptic seizures, antiepileptic drugs, infections or metabolic disturbances. MERS is an encephalopathy syndrome that is typically caused by a viral infection. ${ }^{4}$ The viral infections associated with MERS include mumps, influenza $\mathrm{A}$, adenovirus, dengue virus, varicella-zoster virus, and echovirus $6 .^{4-6}$ The pathophysiology of the disease is unclear. However, the clinical features of the MERS are similar irrespective of the types of microorganism. ${ }^{4}$

We aimed to assess the clinical-radiologic features of a patient with MERS associated with COVID 19 pneumonia.

\section{CASE REPORT}

Informed consent form was obtained from patient for this case report. A 47-year-old male patient was admitted with complaint of personality changes, confusion, and aggression following 5 days of fever.

He had no chronic diseases. Confusion was detected in his neurological examination. There were no meningeal irritation clinically. His deep tendon reflexes were normal and Babinski reflex was flexor. No motor, sensory or cerebellar deficits were detected. His laboratory findings were as follows: white blood cell (WBC): $9.30 \mathrm{~K} / \mu \mathrm{L}$ (3.5-10.5), hemoglobin: $11.2 \mathrm{~g} / \mathrm{dL}$ (13.5-17.5), platelets: $235 \mathrm{~K} / \mathrm{uL}$ (150-450), and vitamin B12: $258 \mathrm{pg} / \mathrm{mL}$ (211-911). Liver-kidney functions test, lipid, creatinine phosphokinase, and electrolytes were normal range. Blood gas analysis and hormonal tests were normal. C-reactive protein elevated $(14.2 \mathrm{mg} / \mathrm{L}$, normal range $0-8 \mathrm{mg} / \mathrm{L})$. Body temperature was $38.3{ }^{\circ} \mathrm{C}$, blood pressure was $140 / 80 \mathrm{mmHg}$, and respiratory rate was $17 / \mathrm{min}$. There was no history of SARS-COV-2 contact. On admission, he had decreased breath sounds at the lung bases. His oxygen saturation was $95 \%$ on room air. He was positive for SARS CoV-2 on nasopharyngeal swab with real-time

Address correspondence to: Fettah Eren, Asst. Prof., M.D. Selcuk University, School of Medicine, Department of Neurology, 42700, Konya, Turkey. Tel: +90 (505) 86041 46, E-mail: dreren42@ hotmail.com

Date of Submission: 13 July 2021; Date of Acceptance: 20 July 2021

https://doi.org/10.54029/2021urw 
polymerase chain reaction (RT-PCR). Thorax computed tomography (CT) showed moderate pulmonary infiltration that was typical for SARSCOV-2 pneumonia (Figure 1. A, B).

Brain magnetic resonance imaging (MRI) showed hyper-intense lesion in the splenium of the corpus callosum on diffusion-weighted imaging and hypo-intense on apparent diffusion coefficient (ADC) mapping (Figure 2. A, B). Electroencephalography showed diffuse slower background activity. He was admitted to the coronavirus unit. The patient rapidly improved with oxygen and prednisone $(1 \mathrm{mg} / \mathrm{kg} / \mathrm{day})$ treatment for five days. His personality changes, confusion, and aggression regressed on $4^{\text {th }}$ day. He left the hospital after 10 days, recovering completely. Repeated brain diffusion weighted imaging and ADC mapping MRI on $15^{\text {th }}$ day was normal with resolution of the lesion in the splenium of the corpus callosum (Figure 2. C, D).

\section{DISCUSSION}

We described a patient with MERS while suffering from pneumonia and psychiatric symptoms associated with SARS-COV-2 infection. This case was diagnosed with SARS-COV-2 infection based on the RT-PCR and thorax computed tomography. In addition, we demonstrated isolated corpus callosum splenium lesion on brain MRI. We speculated that these findings were associated with the COVID-19, with complete recovery symptomatically and in the MRI.

Reversible splenial lesion of the corpus callosum may associated with abnormal electrolytes disorders, organ failures, and administrations of a certain type of drugs. ${ }^{4,7} \mathrm{We}$ assessed the medical and medication history, and found no other cause MERS other than SARSCOV-2 infection. Recent studies reveled that SARS-CoV-2 infection may be etiological factor of meningitis or encephalitis. ${ }^{8}$ Virus-related MERS has been shown to be associated with infection by mumps virus, rotavirus, and influenza virus. ${ }^{4-6}$ However, to the best of our knowledge, MERS induced by coronavirus has been reported only rarely.

Hayashi et al. described the first MERS case associated with SARS-COV-2 infection It was a 75-year-old-male with a history of mild Alzheimer's disease, complaining of leftdominant tremor, walking instability and urinary incontinence. The chest CT scan demonstrated the pulmonary infiltration in inferior lobes. $\mathrm{He}$ did not develop any fever and have a history of contact with SARS-CoV-2. After admission, the patient developed a fever of $39.3^{\circ} \mathrm{C}$. Throat swab RT-PCR test for SARS-CoV-2 was positive.. ${ }^{2}$ Our patient had fever as an initial symptom, and many studies emphasize that MERS may also be associated with fever. ${ }^{9}$ Hayashi et al. reported that their patient had fever at initially. However, fever was observed during the follow-ups. By day 3, the patient became alert, coherent and oriented. He died due to respiratory failure 12 days after the first presentation.2.

Chauffier et al. described another MERS case associated with SARS-COV-2 infection. This was a 47-year-old man with no medical

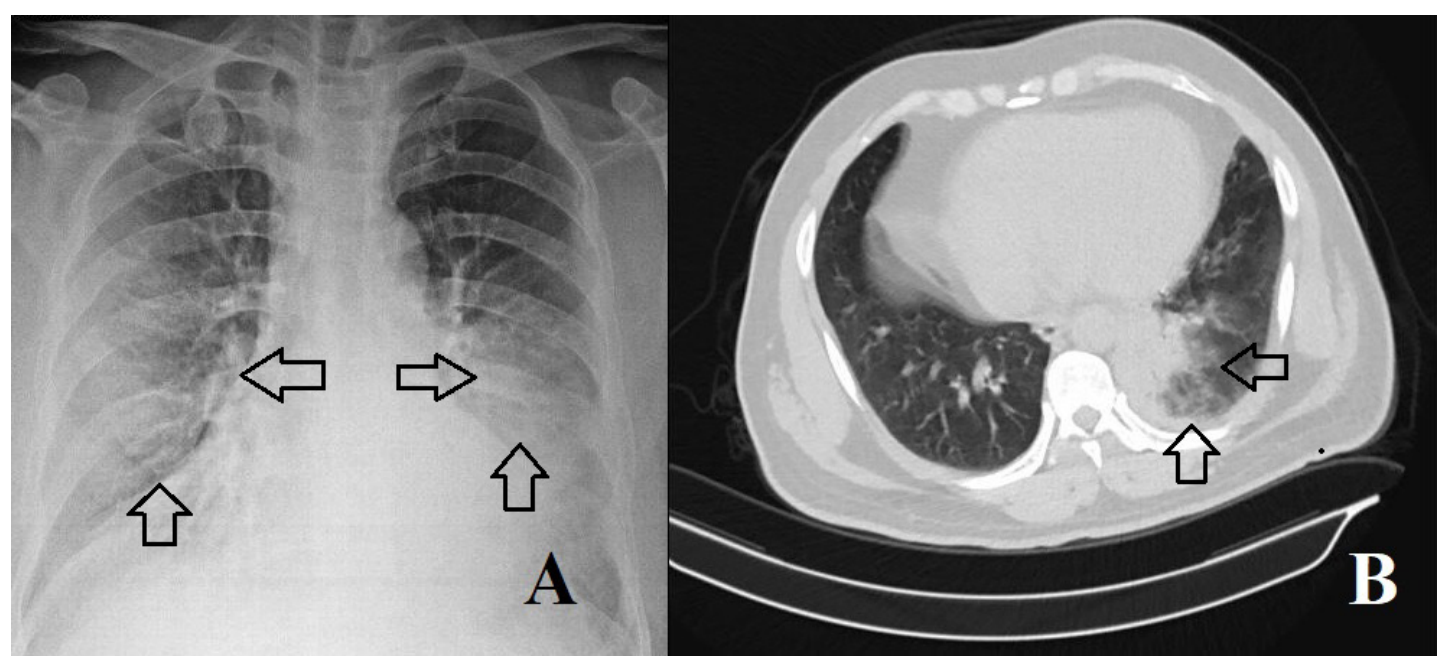

Figure 1. A. Posterior-anterior X-ray, B. Thorax computed tomography: pulmonary infiltration, typical for SARSCOV-2 pneumonia 


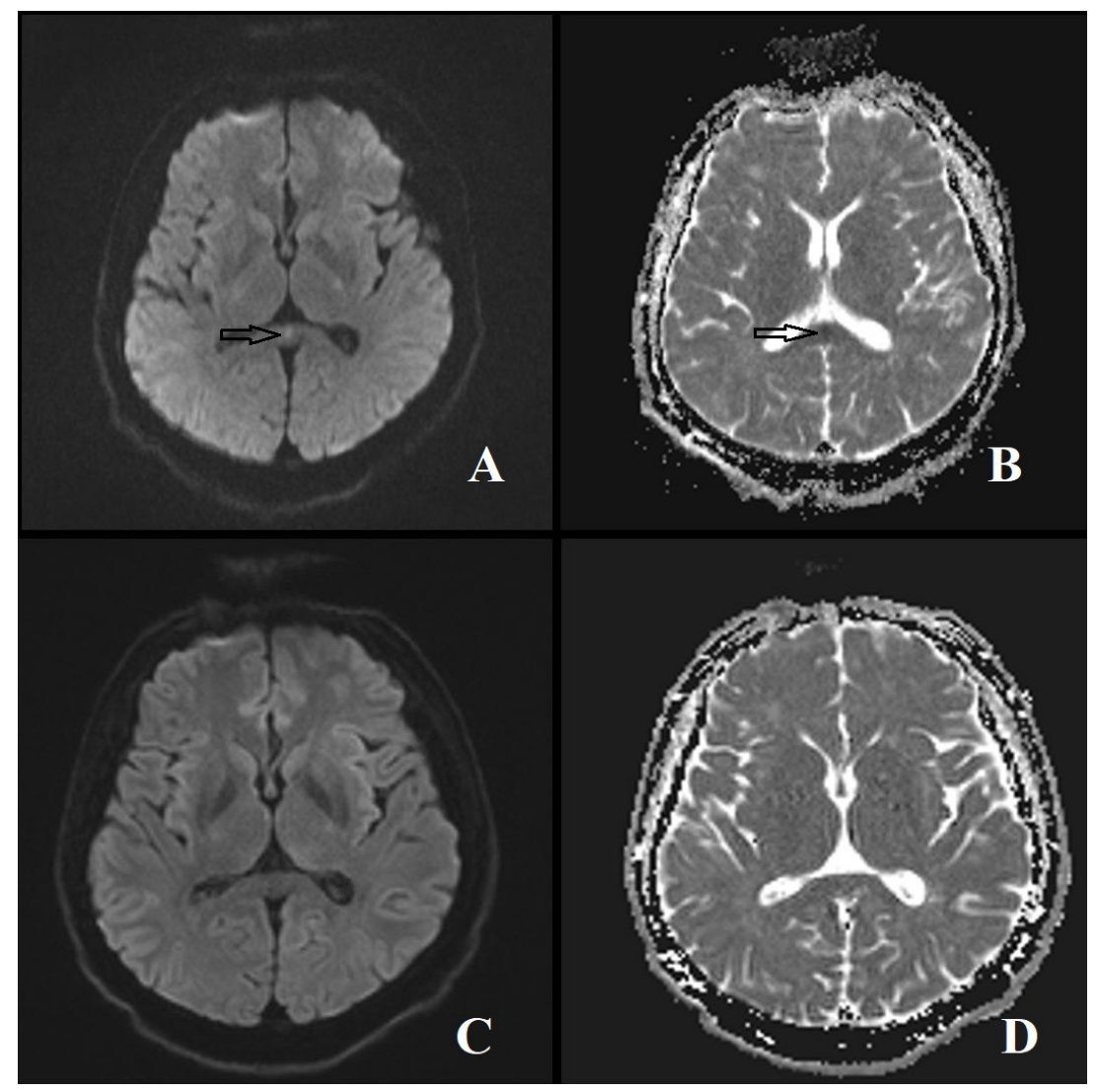

Figure 2. Brain magnetic resonance imaging A. Diffusion-weighted imaging B. Apparent diffusion coefficient (ADC) mapping (A, B: At the time of presentation; $C, D: 15^{\text {th }}$ day)

A, B: A lesion in the splenium of the corpus callosum C, D: Resolution of the lesion in the splenium of the corpus callosum

history or treatment with febrile cough and headache symptoms for 15 days. He presented with normal body temperature. Neurological assessment revealed inattention, disinhibition, and logorrhea. Nasopharyngeal swab was positive for SARS-CoV-2 . Moderate hyponatremia was detected. Moderate lung damage typical of SARS-CoV-2 pneumonia with pulmonary CT. Cerebrospinalfluid (CSF) examination was normal for cell counts and for protein and glucose levels and was negative for SARS-CoV-2. The patient improved without any treatment other than oxygen and left the hospital on $7^{\text {th }}$ days. ${ }^{3}$ MERS is associated with hyponatremia. ${ }^{4}$ It is not possible to evaluate the association of MERS with hyponatremia and/or SARS-COV-2 in this patient. Our patient did not have electrolyte disturbances. Lumbar puncture was not performed, since our patient was considered high probability of prediagnosis of MERS.

Bektaş et al. reported two children with multisystem inflammatory syndrome related to
SARS-CoV-2. Encephalopathy was the main symptom. Both of the children showed a rapid clinical and radiological recovery; both had initial fever, but no electrolyte imbalance was detected. Cerebrospinal fluid examination was not performed in these two patients. ${ }^{10}$

In conclusion, we described a patient with MERS associated with SARS CoV-2 infection and its clinical and radiological features. Complete resolution of corpus callosum lesion and recovery of encephalopathy associated with SARS-COV-2 were similar to previous cases.

\section{DISCLOSURE}

Financial support: None

Conflict of Interest: Nothing to declare

\section{REFERENCES}

1. Bridwell R, Long B, Gottlieb M. Neurologic complications of COVID-19. Am J Emerg Med 2020; 38:1549.e3-1549.e7. 
2. Hayashi M, Sahashi Y, Baba Y, Okura H, Shimohata T. COVID-19-associated mild encephalitis/ encephalopathy with a reversible splenial lesion. $J$ Neurol Sci 2020; 415:116941.

3. Chauffier J, Poey N, Husain M, et al. First Case of Mild Encephalopathy with Reversible Splenial Lesion in SARS-CoV-2 Infection. Infect Dis Now 2021; 51:99-101.

4. Tada H, Takanashi J, Barkovich AJ, et al. Clinically mild encephalitis/encephalopathy with a reversible splenial lesion. Neurology 2004; 63(10):1854-8.

5. Fong CY, Khine MM, Peter AB, Lim WK, Rozalli FI, Rahmat K. Mild encephalitis/encephalopathy with reversible splenial lesion (MERS) due to dengue virus. J Clin Neurosci. 2017; 36:73-5.

6. Masiello E, Gatto A, Lazzaresc I, Rigante D, Mariotti P, Valentini P. Mild encephalopathy with reversible splenial lesion associated with echovirus 6 infection: a case report and review of the literature. Turk $J$ Pediatr 2020; 62:293-309.

7. Garcia-Monco JC, Cortina IE, Ferreira E, et al. Reversible splenial lesion syndrome (RESLES): what's in a name?. J Neuroimaging 2011; 21:e1-e14.

8. Moriguchi T, Harii N, Goto J, et al. A first case of meningitis/encephalitis associated with SARSCoronavirus-2. Int J Infect Dis 2020; 94:55-58.

9. Xu W, Zhou Y, Jiang Y, Zhang L, Tang Q. Clinical and Magnetic Resonance Imaging Features of Reversible Splenial Lesion Syndrome in Adults: A Small Case Series. Eur Neurol 2019; 82:86-92.

10. Bektaş G, Akçay N, Boydă̆ K, Şevketoğlu E. Reversible splenial lesion syndrome associated with SARS-CoV-2 infection in two children. Brain Dev 2021; 43:230-3 